\title{
BARYON SPECTROSCOPY ON THE LATTICE
}

\author{
ROBERT G. EDWARDS \\ Jefferson $L a b$ \\ 12000 Jefferson Avenue Newport News, Virginia 23606, USA \\ E-mail: edwards@jlab.org
}

Recent lattice QCD calculations of the baryon spectrum are outlined.

\section{Introduction}

Quantum Chromodynamics (QCD) provides an excellent description of nature; however, the theory suffers from divergences that must be removed to render it finite. Lattice QCD provides an apriori non-perturbative regularization of QCD that makes it amenable to analytic and computational methods. No model assumptions other than QCD itself are needed to formulate the theory. This review surveys the rapidly evolving work in using Lattice QCD for calculations of baryon spectroscopy. Along the way, sources of systematic uncertainties in calculations are described and future directions are outlined.

\subsection{Regularization of $Q C D$ on a lattice}

As the starting point for lattice QCD, the path integral formulation in Euclidean space is usedt. The usual continuous space-time of 4-dimensional continuum QCD are approximated with a discrete 4-dimensional lattice, with derivatives approximated by finite differences. Quarks are put on sites, gluons on links. Gluons are represented as $3 \times 3$ complex unitary matrices $U_{\mu}(x)=\exp \left(i g a A_{\mu}(x)\right)$ elements of the group $\mathrm{SU}(3)$ with vector potential $A_{\mu}(x)$, coupling $g$, and lattice spacing $a$. The vacuum expectation value of operators involves path integration over gauge and fermion fields

$$
\begin{gathered}
\langle O(U, \psi, \bar{\psi})\rangle=\frac{1}{Z} \int d U_{\mu} d \bar{\psi} d \psi O(U, \psi, \bar{\psi}) e^{-S_{G}(U)+\bar{\psi} M(U) \psi} \\
\rightarrow \frac{1}{Z^{\prime}} \int d U_{\mu} O\left(U, M^{-1}(U)\right) \operatorname{det}(M(U)) e^{-S_{G}(U)} .
\end{gathered}
$$

The Gaussian integration over the anti-commuting fermion fields $\psi$ resulted in the $\operatorname{det}(M(U))$ and $M^{-1}(U)$ factors with $M(U)$ a lattice form of the Dirac operator. The gauge action $S_{G}(U)$ approximates the Yang-Mills action of the continuum. The quenched approximation neglects the fermion determinant.

baryons'2002: submitted to World Scientific on November 14, 2018 
The choice of working in Euclidean space resulted in no factors of $i$ in the exponent multiplying the gauge and fermion actions. The path integral therefore resembles a 4-dimensional statistical mechanical model making it amenable to analytic as well as Monte Carlo methods for evaluation.

Numerical predictions from lattice QCD are in principle exact (to some precision) after systematic errors are controlled. The statistical uncertainties go like $1 / \sqrt{N}$ for $\mathrm{N}$ configurations of gauge fields in a Monte Carlo ensemble average. Systematic uncertainties include: (1) Finite volume - the lattice box must hold a hadron state, typically a lattice size of $L \sim 2 \mathrm{fm}$ or more is needed. Several pion Compton wavelengths are needed $m_{\pi} L \sim 4$. (2) Chiral extrapolations - calculations with small quark masses are expensive - extrapolate observables to physical quark mass region (delicate!). (3) Discretization effects: inherent $\mathcal{O}(a)$ or $\mathcal{O}\left(a^{2}\right)$ lattice uncertainty. One must extrapolate to continuum limit $(a \rightarrow 0)$ to recover physical quantities.

\section{Confinement and Model Predictions - Static Quark Potentials}

A particularly useful rôle of lattice QCD is model testing. There is significant recent activity in the study of 3 quark potentials which provide phenomenological insight into the forces inside a baryon. By gauge invariance, the quarks must be joined by 3 glue strings. These strings meet at a "gluon junction", which has been conjectured to be a non-perturbative excitation of the QCD vacuum 2 . What is the area law behavior? One can test two ansätze.

The $Y$-ansatz predicts the potential grows linearly like $V_{q q q} \propto \sigma_{q q} L_{Y}$ where $L_{Y}$ is the minimal length of the 3 flux tubes necessary to join the 3 quarks at the Steiner point. It is derived from strong coupling arguments 3 , and is consistent with the dual superconductivity confinement scenario.

At large distances, the $\Delta$-ansatz predicts instead that the potential grows linearly with the perimeter $L_{\Delta}$ of the quark triangle, e.g. $V_{q q q} \propto \sigma_{q \bar{q}} L_{\Delta} / 2$. It is derived from a model of confinement by center vortices using a topological argument 1 .

There is controversy as to which ansaty 0 lids. Recent work 6 claims that at short distances the potential approaches the $\Delta$-ansatz but rises like the $Y$-ansatz at large distances. Departures from the $\Delta$-ansatz appear above $d_{q q} \sim 0.7 \mathrm{fm}$ hence the $\Delta$ model is more appropriate inside a hadron. However, recently Simonovd claims there is a field strength depletion near the $Y$-junction which lowers the potential and could disguise the true behavior. Tests using adjoint sources could help reconcile the various claims.

baryons'2002: submitted to World Scientific on November 14, 2018 


\section{Hadron Spectrum}

\subsection{Chiral Symmetry}

As mentioned before, for accurate lattice calculations systematic uncertainties need to be controlled. The discretization of the Dirac operator has been particularly troublesome since lattice QCD's inception and can significantly affect continuum and chiral extrapolations. The "doubling" problem is easily demonstrated by examining the lattice momentum representation of the free Dirac operator, namely $\sum_{\mu} \gamma_{\mu} \partial_{\mu} \rightarrow \frac{i}{a} \sum_{\mu} \gamma_{\mu} \sin \left(a p_{\mu}\right)$. The propagator has additional zeros at the momentum corners, e.g. $a p_{\mu}=0, \pi$ so there are 16 species of fermions in general. Originally, Wilson lifted the doublers by adding a Laplacian term that breaks chiral symmetry. In fact, the Nielson-Ninomia no-go theorems state one cannot avoid both doubling and chiral symmetry breaking with a local, hermitian action analytic in the gauge fields. This major theoretical problem has been solved with the recent advent of chiral fermion actions (e.g., Domain-Wall or Overlap fermions) and their use is crucial for matrix elements. How important is chiral symmetry for spectroscopy studies?

Renormalization theory tells us that breaking a symmetry leads to induced quantum terms in an action. The Wilson fermion action has $\mathcal{O}(a)$ scaling from the breaking of chiral symmetry. One can add a dimension 5 operator (hyper-fine term) and rigorously improve scaling from $\mathcal{O}(a)$ to $\mathcal{O}\left(a^{2}\right)$. Scaling violations are dramatically reduced - mostly from improving chiral symmetry. Scaling violations are comparable with chiral fermion formulations. The conclusion is that chiral symmetry is important for accurate spectrum calculations 9 at comparatively heavy quark masses. However, the benefits of chiral fermion actions with exact chiral symmetry are now being dramatically demonstrated as near physical quark masses are approached as will be shown below.

\subsection{Quenched Pathologies in Hadron Spectrum}

Clearly, precisely controlled lattice calculations come with the inclusion of the fermion determinant. However, because of their vastly reduced computational cost quenched calculations are quite prevalent and one can gain important phenomenological insight into QCD, but the potentially large systematic errors induced in this approximation should be carefully ascertained. Suppressing the fermion determipgnt leads to well known pathologies as studied in chiral perturbation theory 11 . There are missing vacuum contributions to

baryons'2002: submitted to World Scientific on November 14, 2018 
the disconnected piece of singlet correlators

$$
\begin{gathered}
\left\langle\bar{\psi}(x) \gamma_{5} \psi(x) \bar{\psi}(y) \gamma_{5} \psi(y)\right\rangle=\left\langle\operatorname{Tr}\left[\gamma_{5} G(x, y) \gamma_{5} G(y, x)\right]_{c, s}\right\rangle \\
-N_{f}\left\langle\operatorname{Tr}\left[\gamma_{5} G(x, x)\right]_{c, s} \operatorname{Tr}\left[\gamma_{5} G(y, y)\right]_{c, s}\right\rangle .
\end{gathered}
$$

These effects are manifested in the $\eta^{\prime}$ propagator missing vacuum contributions with new double pole divergences arising of the form

$$
\int \frac{d^{4} p}{(2 \pi)^{4}} e^{i p \cdot x}\left\langle\operatorname{Tr} \gamma_{5} G(x, x) \operatorname{Tr} \gamma_{5} G(0,0)\right\rangle=f_{P} \frac{1}{p^{2}+m_{\pi}^{2}} m_{0}^{2} \frac{1}{p^{2}+m_{\pi}^{2}} f_{p}+\ldots
$$

How dramatic are these quenched effects and to what extent do they affect the extraction of physical observables? One idea is to incorporate knowledge of quenched divergences in calculations and then attempt to extract useful information.

\subsection{Decay in the Quenched Approximation}

The $m_{0}^{2}$ in Eq. (11) is the mass shift needef to recover the pseudoscalar singlet mass from the non-singlet pion. In 10 , the unique piece of the $\eta^{\prime}$ correlator - the hairpin - was computed directly. The parameter $m_{0}^{2}$ was extracted and one sees the lattice data is well described by the $\chi \mathrm{PT}$ prediction. With the shift, the $\eta^{\prime}$ mass (at non-zero lattice spacing) is determined to be $820(30) \mathrm{MeV}$ with possibly large $\mathcal{O}(a)$ scaling uncertaintities. A recent Domain Wall calculation 12 gives $940(4) \mathrm{MeV}$.

Further dramatic behavior is seen in the isotriplet scalar particle $a_{0}$. There is an $\eta^{\prime}-\pi$ intermediate state with missing contributions in the quenched approximation as shown in Fig. (11). In fact, the $a_{0}$ correlator goes negative - a clear sign of violations of unitarity 13 . From $\chi \mathrm{PT}$, one can construct the $a 0$ correlator by including couplings between $\eta^{\prime}-\pi$ states and rescattering states which can be resummed. The lightest $a 0$ correlator is fairly well described by a 1-loop resummed bubble term with $\eta^{\prime}$ mass insertion fixed A mass $m_{a_{0}}=1.34(9) \mathrm{GeV}$ was found. The new Domain Wall calculation 12 gives $m_{a_{0}}=1.04(7) \mathrm{GeV}$. The latter results does not exclude the possibility of $a 0(980)$ being a $\bar{q} q$ state.

\subsection{Quenched and Full QCD Hadron Spectrum}

The quenched low-lying hadron spectrum has been extensively studied by the CPPACS collaboration using Wilson fermions 14 . Masses were computed at four lattice spacings and extrapolated to the continuum limit. Lattice sizes

baryons'2002: submitted to World Scientific on November 14, 2018 


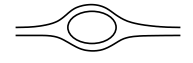

(a)

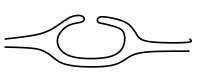

(b)

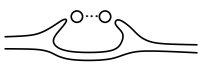

(c)

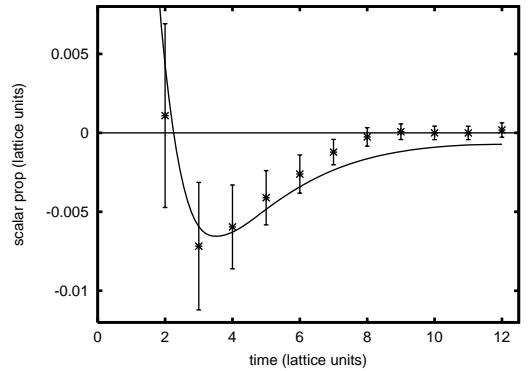

Figure 1. Left: contributions to the $a_{0}$ propagator from an $\eta-\pi$ intermediate state. Right: comparison of scalar $a_{0}$ propagator with the bubble sum formula fitted to the interval $\mathrm{t}=1-6$ (Ref.13)

ranged up to $64^{3} \times 112$ for a $3.2 \mathrm{fm}$ box. At each lattice spacing, the lightest pseudoscalar mass obtained was about 500 to $600 \mathrm{MeV}$. Hadron masses were extrapolated in the quark mass via an ansätz motivated by the quenched $\chi \mathrm{PT}$ prediction

$$
\begin{aligned}
& m_{P S, 12}^{2}=A\left(m_{1}+m_{2}\right)\left\{1-\delta\left[\ln \left(2 A m_{1} / \Lambda_{\mathrm{X}}^{2}\right)\right]\right. \\
& \left.\quad+m_{2} /\left(m_{2}-m_{1}\right) \ln \left(m_{2} / m_{1}\right)\right\}+B\left(m_{1}+m_{2}\right)^{2}+\mathrm{O}\left(m^{3}\right) \\
& m_{H}\left(m_{P S}\right)=m_{0}+C_{1 / 2} m_{P S}+C_{1} m_{P S}^{2}+C_{3 / 2} m_{P S}^{3}, \quad C_{1 / 2} \propto \delta .
\end{aligned}
$$

The constants $A, B$, and $C_{i}$ are fixed in the fits. Quenching effects were more clearly seen in the pseudoscalar channel. The calculation shows the basic hadron spectrum is well determined even in the quenched approximation to within $10 \%$ accuracy. The computational cost was roughly 50 Gigaflop-years.

A subsequent two-flavor dynamical calculation was made with four quark masses at 3 lattice spacings 15 . Box sizes range up to about $2.5 \mathrm{fm}$. In the meson sector, the results are consistent with the original quenched calculations and now agree to within $1 \%$ of experiment. Systematic deviations of the quenched calculation from experiment are seen demonstrating that sea quark effects are important. The vector meson masses are increased after unquenching. This increased hyperfine splitting is consistent with the qualitative view that the spin-spin coupling in quenched QCD is suppressed compared to full QCD due to a faster running of the coupling constant.

In the baryon sector, two-flavor dynamical sea quark effects are not as apparent. As seen in Fig. (2)). The $N$ and $\Delta$ masses are higher than experiment, but other masses are consistent. With only a $2.5 \mathrm{fm}$ box, finite-volume effects could well be large. Another concern is that the octet and decuplet chiral extrapolations have many parameters resulting in possibly underestimated er- 

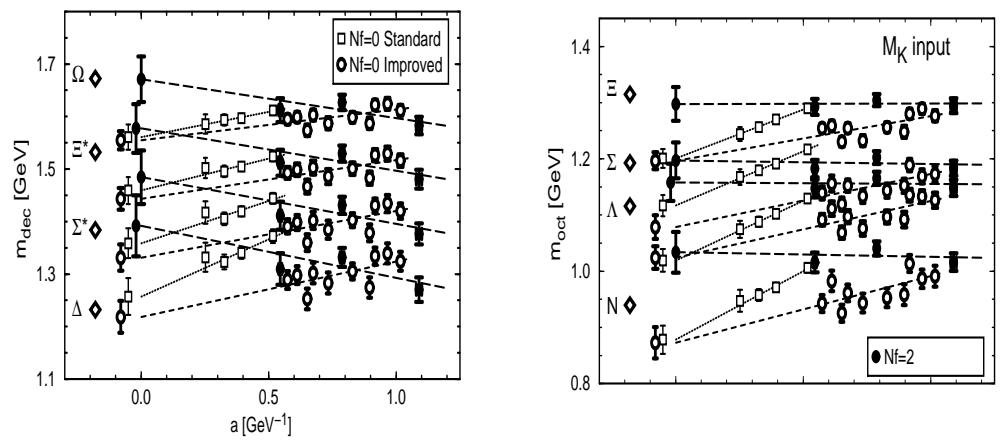

Figure 2. Baryon masses in two-flavor (filled symbols) md quenched (open symbols) QCD. Both graphs have the same lattice spacing scale. (Ref.15).

rors and will be discussed more next. However, this is a significant calculation involving roughly one Teraflop-year of computations and well demonstrates the efficacy of lattice methods.

\subsection{Improved Chiral Extrapolations}

The Adelaide group has been extensively studying higher order $\chi \mathrm{PT}$ effects on hadronic quantities 16 . The basic upshot is that the naive chiral extrapolations in use are just too naïve! In particular, they incorporate leading non-analytic behavior from heavy baryon $\chi \mathrm{PT}$ arising from $B \rightarrow B^{\prime} \pi \rightarrow B$ intermediate states with $B=N, \Delta$

$$
M_{B}=\alpha_{B}+\beta_{B} m_{\pi}^{2}+\Sigma_{B}\left(m_{\pi}, \Lambda\right)
$$

where $\Sigma_{B}$ is a self-energy term. The coefficient of the $m_{\pi}^{3}$ term is actually known analytically in contrast to Eq. (2). The basic argument is since $\chi \mathrm{PT}$ has a zero radius of convergence (or certainly not a well defined radius), a simple leading order approximation to Eq. (3) is quite a bad approximation at moderate $m_{\pi}$. They use a simple regularization of the self-energy term.

Fig. (3) shows a comparison of a recent quenched and $2+1$ dynamical calculation of the low lying hadron spectrum for a variant of staggered fermions at the same (physical) lattice spacing 16 . The various lines are from fits using Eq. (3). One can see that the self energy term becomes significant for small lattice quark masses. The intriguing result is that the fit parameters $\alpha_{B}$ and $\beta_{B}$ agree very well between the quenched and dynamical calculations. This 

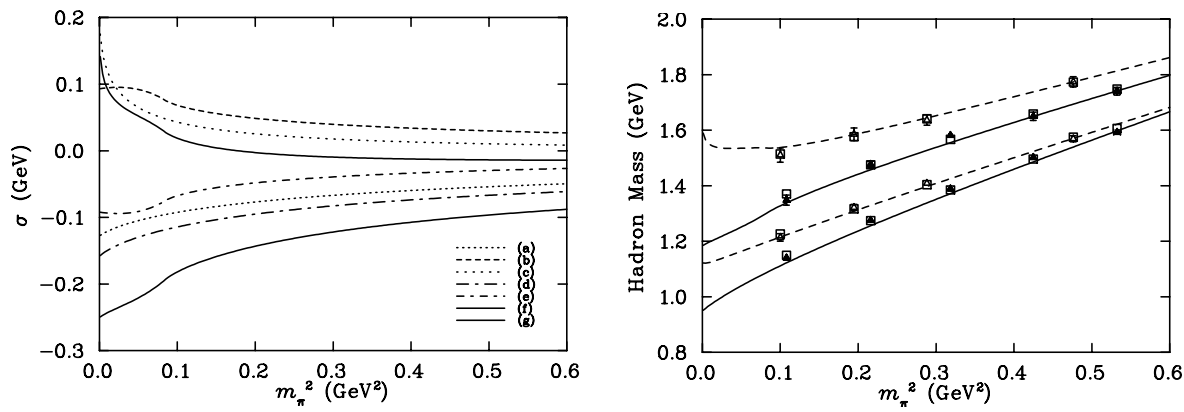

Figure 3. Left: contributions from various intermediate states to the quenched and unquenched self-energy term $\Sigma$. Right: fit (open squares) to lattice data - quenched (open $\triangle$ ) and dynamical (filled $\triangle$ ) with adjusted self-energy expressions accounting for finite volume and lattice spacing artifacts. The continuum limit of quenched (dashed lines) mid dynamical (solid lines) are shown. The lower curves are for $N$ and upper for $\Delta$. (Ref. 16.)

result can be used to justify the claim that the dominant effects of quenching is attributed to first order meson loop corrections.

While quite intriguing it is fair to say there is some controversy over these results. At issue is the concern that once one uses any model to directly interpret lattice results, one has lost predictably. However, in defense once one used a chiral extrapolation at all one has chosen a model. Ultimately, the Adelaide's group work has shown that their is interesting structure in the "pion cloud" around a hadron and going to light quark masses is essential.

\subsection{Excited Baryons}

Understanding the $N^{*}$ spectrum gives vital clues about the dynamics of QCD and hadronic physics. Some open mysteries are what is the nature of the Roper resonance? Why is the ordering of the lowest-lying states - the positive and negative parity states - inverted between the $N, \Delta$ and $\Lambda$ channels?

The history of lattice studies of excited baryons is quite brief. Recently, new calculations are starting to appear using improved gauge and fermion actions. The nucleon channel is the most studied and work has focussed on two independent local interpolating fields

$$
N_{1}=\varepsilon_{i j k}\left(u_{i}^{T} C \gamma_{5} d_{j}\right) u_{k}, \quad N_{2}=\varepsilon_{i j k}\left(u_{i}^{T} C d_{j}\right) \gamma_{5} u_{k} .
$$

Both interpolating fields couple to positive and negative parity states, so in practice parity projection techniques are used. Making the lattice anisotropic with finer discretization in time allows the behavior of the correlators to be 

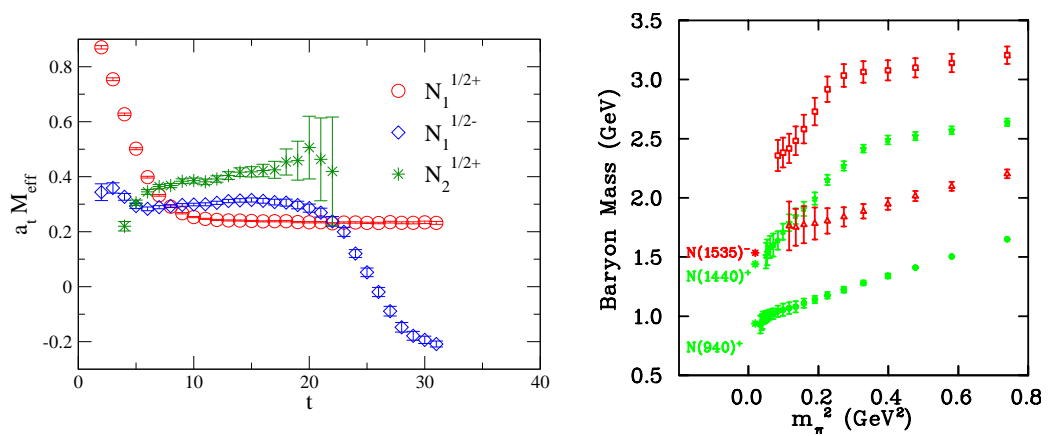

Figure 4. Left: effective masses for correlators with fields Eq. (1) corresponding to the nucleon (circles), its parity partner (diamonds) and te patively the lowest positive parity excitation (cross) with an anisotropic clover actiop_Ref.17). Right: masses in physical units obtained with the isotropic Overlap action (Ref.18). Solid symbols denote $N\left(\frac{1}{2}^{+}\right)$states: ground $(\bullet)$ and 1st-excited $(\star)$. Empty symbols denote $N\left(\frac{1}{2}^{-}\right)$states: lowest $(\triangle)$ and 2nd lowest $(\square)$. The experimental points $(*)$ are taken from PDG.

examined over many more time slices than on isotropic lattices. Additional tuning of the fermion action is needed to recover hypercubic symmetry.

The left side of Fig. (雨) shows the effective mass for the nucleon and its parity partner on an anisotropic lattice using the clover action 17 - an action improved to have reduced discretization uncertainties. Long plateaus (clean extraction of a mass) are seen demonstrating the efficacy of the method. For this quark mass, there appears to be the expected ordering of the states $N^{* 1 / 2+}>N^{1 / 2-}>N^{1 / 2+}$. However, there is difficulty in approaching small quark masses, and the mass extracted with the $N_{2}$ operator appears too large.

A recent quenched calculation 18 using Overlap fermions (a chiral fermion action) going to much smaller quark masses reveals a dramatic decrease in the nucleon masses extracted only with the $N_{1}$ interpolating field as seen in the right side of Fig. (4). The authors claim the apparent crossing of the first excited $N^{1 / 2}$ and lowest $N^{1 / 2-}$ states is the demonstration of the physically correct ordering of states. However, it is also possible that at such light quark masses a decay threshold has been crossed and the mass observed is affected by missing dynamical effects via the mechanisms described in Sec-3.3. If so, a finite volume check via shrinking the lattice box can reveal this. It is clear lattice calculations are really beginning to probe interesting excited state phenomena. With judicious use of finite volume techniques, physically relevant mass information can be extracted. 


\section{Conclusions}

First generation lattice calculations of excited baryon spectroscopy are appearing. State of the art calculations require roughly 100 Gigaflop-year in quenched QCD and roughly 1 to 10 Teraflop-years in full QCD. The required resources are not available to the US lattice community. The Dept. of Energy's SciDAC program is addressing this shortcoming and a large effort is ongoing in the U.S. to meet future computational needs.

\section{Acknowledgments}

RGE was supported by DOE contract DE-AC05-84ER40150 under which the Southeastern Universities Research Association (SURA) operates the Thomas Jefferson National Accelerator Facility (TJNAF).

\section{References}

1. I. Montvay and G. Münster, Quantum Fields on a Lattice, (Cambridge Univ. Press, 1994).

2. D. Kharzeev, Phys. Lett. B 378, 238 (1996).

3. N. Isgur and J. Paton, Phys. Rev. D 31, 2910 (1985).

4. J. M. Cornwall, Phys. Rev. D 54, 6527 (1996).

5. T. T. Takahashi, et.al., Phys. Rev. D 65, 114509 (2002).

6. C. Alexandrou, P. De Forcrand and A. Tsapalis, hep-lat/0209062.

7. D.S. Kuzmenko, Yu.A. Simonov, hep-ph/0202277.

8. D.B. Kaplan, Phys. Lett. B288, 342 (1992). R. Narayanan and H. Neuberger, Nucl. Phys. B443, 305 (1995).

9. R.G. Edwards, U.M Heller, T.R. Klassen, Phys. Rev. Lett. 80, 3448 (1998).

10. B. Bardeen, et.al., Phys. Rev. D 62, 114505 (2000).

11. C. Bernard and M. Golterman, Phys. Rev. D 46, 853 (1992). S. Sharpe, Phys. Rev. D 46, 3146 (1992).

12. S. Prelovsek and K. Orginos, hep-lat/0209132.

13. B. Bardeen, et.al., Phys. Rev. D 65, 014509 (2002).

14. S. Aoki, et.al., Phys. Rev. Lett. 84, 238 (2000).

15. A. Ali Khan, et.al., Phys. Rev. D 65, 054505 (2002).

16. R.D. Young, et.al., hep-lat/0111041.

17. R.G. Edwards, U. Heller, D. Richards, proceedings of Lattice 02.

18. F.X. Lee, et.al., hep-lat/0208070. 